\title{
Article \\ A Randomised Controlled Trial of Ice to Reduce the Pain of Immunisation-The ICE Trial
}

\author{
Yashodha Ediriweera *, Jennifer Banks, Leanne Hall (D) and Clare Heal (D) \\ Mackay Clinical School, College of Medicine and Dentistry, James Cook University, 475 Bridge Road, \\ Mackay, QLD 4740, Australia; banks.jennifer.jcu@gmail.com (J.B.); leanne.hall@jcu.edu.au (L.H.); \\ clare.heal@jcu.edu.au (C.H.) \\ * Correspondence: yashodha.ediriweera@my.jcu.edu.au
}

check for updates

Citation: Ediriweera, Y.; Banks, J.; Hall, L.; Heal, C. A Randomised Controlled Trial of Ice to Reduce the Pain of Immunisation-The ICE Trial. Trop. Med. Infect. Dis. 2021, 6, 158. https://doi.org/10.3390/ tropicalmed6030158

Academic Editor: John Frean

Received: 1 August 2021

Accepted: 24 August 2021

Published: 28 August 2021

Publisher's Note: MDPI stays neutral with regard to jurisdictional claims in published maps and institutional affiliations.

Copyright: (c) 2021 by the authors. Licensee MDPI, Basel, Switzerland. This article is an open access article distributed under the terms and conditions of the Creative Commons Attribution (CC BY) license (https:// creativecommons.org/licenses/by/ $4.0 /)$.

\begin{abstract}
Background and objectives: vaccine injections are a common cause of iatrogenic pain and anxiety, contributing to non-compliance with scheduled vaccinations. With injection-related pain being recognised as a barrier to vaccination uptake in both adults and children, it is important to investigate strategies to effectively reduce immunisation pain. This prospective randomised controlled trial investigated the effects of applying an ice pack on vaccine-related pain in adults. Methods: medical students receiving the flu vaccination were randomised to receive an ice pack (intervention) or placebo cold pack (control) at the injection site for $30 \mathrm{~s}$ prior to needle insertion. Immediate post-vaccination pain (VAS) and adverse reactions in the proceeding $24 \mathrm{~h}$ were recorded. Results: pain scores between the intervention $(n=19)$ and control groups $(n=16)$ were not statistically significant (intervention: median pain VAS $=7.00, \mathrm{IQR}=18$; control: median pain $\mathrm{VAS}=11, \mathrm{IQR}=14$ $(p=0.26)$. There were no significant differences in the number of adverse events between the two groups (site pain $p=0.18$; localised swelling ( $p=0.67$ ); bruising $p=0.09$; erythema $p=0.46$ ). Discussion: ice did not reduce vaccination-related pain compared to cold packs. COVID-19 related restrictions impacted participant recruitment, rendering the study insufficiently powered to draw conclusions about the results.
\end{abstract}

Keywords: primary health care; cold therapy; analgesia; general practice; vaccinations

\section{Introduction}

Vaccine injections are the most common cause of iatrogenic pain in childhood, and can cause anxiety in adults [1,2]. A 2003 survey reported $21 \%$ to $90 \%$ of adults experienced anxiety about pain associated with needle-based procedures [3]. In severe cases, this leads to injection phobia and subsequent syncopal attacks [4,5]. Vaccination pain is now a recognised adverse effect, and adequate pain management strategies should be incorporated into every vaccination [6].

The physiological mechanisms for analgesic effects of cold temperature are multitheoretical. The gate control theory of pain by Melzack and Wall in 1965 theorised those noxious inputs relayed by small, myelinated $A-\delta$ fibres and unmyelinated $C$ fibres are inhibited by non-noxious stimuli concurrently conducted by A- $\beta$ fibres to the dorsal horn. Consequently, the intensity of the ascending pain stimuli is decreased [7]. Kunesch et al. demonstrated that effects of skin cooling were more pronounced on $C$ fibres compared to A fibres, leading to reduced autonomic response and pain sensation [8]. Another theory, based on animal models, is activation of the analgesic descending pathway stimulated by cold which produces opiate-like peptides [9]. In general, ice packs ( $<5$ degrees Celsius) are known to reduce the temperature of skin and tissues to up $2 \mathrm{~cm}$ deep and induce almost immediate localised skin analgesia $[10,11]$. A previous study demonstrated that a $30 \mathrm{~s}$ application of ice reduced pain levels during tetanus vaccination in adults [12]. A 100C cold pack requires a minimum 20 min application to achieve a similar decrease in skin temperature, and therefore is unable to provide rapid skin analgesia [11]. 
Literature on use of cooling for injection-related pain is sparse, with studies showing conflicting results. The majority of cooling studies have investigated vapocoolants for their vaccination-related pain mitigation properties, with only three out of 13 randomised controlled trials (RCTs) using ice as a cooling method [13]. Only one of these was conducted in an adult population, [12] thus necessitating further research.

This prospective RCT investigates the effect of application of an ice pack $\left(0^{\circ} \mathrm{C}\right)$ to the site of injection prior to immunisation on flu vaccine-related pain levels. The secondary objective was to record adverse reactions to the cooling packs or flu vaccine.

\section{Methods}

\subsection{Study Design and Participants}

This multicentre, double-blinded prospective randomised controlled trial was registered with the Australian and New Zealand Clinical Trials Registry (ANZCTR)- ACTRN12621000064808. The published study protocol provides full methodological details. [14] This double blinded RCT was designed with involvement from the James Cook University Medical Student Association (JCUMSA). Medical students over the age of 18 years attending the annual JCUMSA flu vaccination clinics were invited to participate. Those with a known history of cold anaphylaxis, serious immunisation reactions or allergy to influenza vaccines were excluded. An independent research officer was responsible for recruitment and consenting of medical students to prevent potential coercion to participate. A detailed summary of the project, including potential risks, was provided and all participants gave written informed consent prior to the study. This research conforms to the Declaration of Helsinki and was approved by the James Cook University Human Research Ethics committee (H7871).

\subsection{Randomisation and Blinding}

Randomisation was performed at the participant level in a 1:1 ratio. The random sequence was generated from a computer-generated number table. Allocations were concealed using sealed, numbered, tamperproof opaque envelopes until participants were consented to the trial to minimise potential selection or confounding bias. Members of the research team involved in assessment or administration of vaccines had no role in the assignment process. Participants were blinded to treatment allocation. Ice packs and placebo cold packs were stored separately in identical coolers to blind the doctor administering vaccines.

\subsection{Intervention Procedure}

All participants had an ice pack (intervention), or cold pack (control) applied to the site of injection for $30 \mathrm{~s}$ prior to the administration of the influenza vaccine. A vaccination protocol, modelled on the Royal Australian College of General Practitioners (RACGP) immunisation and influenza prevention guidelines, was used to standardise management across both study arms $[15,16]$. Influenza vaccines were administered immediately after removal of the ice or cold pack and all participants received a standard set of verbal and written post-immunisation instructions.

Modelled on previous studies investigating adult vaccination pain, our study utilised a $100 \mathrm{~mm}$ Visual Analogue Scale (VAS) for measuring post-immunisation pain [12,17]. A $15 \mathrm{~mm}$ difference on the VAS was selected as the minimum clinically significant difference for our study [18]. Further, the VAS has a standard deviation of $26 \mathrm{~mm}$. As such, a sample size of 45 per group was calculated to achieve $80 \%$ power with $p=0.05$ [19].

\subsection{Data Collection}

Baseline data included age, sex, ethnicity and past medical history. Participants recorded their immediate post-immunisation pain on a $100 \mathrm{~mm}$ VAS with $0 \mathrm{~mm}$ being no pain and $100 \mathrm{~mm}$ being the highest pain [18-20]. 
Secondary outcome measures included adverse reactions to the vaccination process, such as anaphylaxis, skin irritation, redness or contact dermatitis. Participants were advised to assess the injection site $24 \mathrm{~h}$ post-vaccination, complete a self-assessment form and return it to the clinic within $48 \mathrm{~h}$ of vaccination. Additionally, the vaccination sites were to be assessed for evidence of side effects if patients present opportunistically or for review due to side effects.

\subsection{Statistical Analysis}

The primary analysis was by intention-to-treat of all randomised participants. The individual person was considered as the unit of analysis. Significance was considered $p<0.05$. Baseline data across the two groups were assessed for marked differences. Numerical data were described as median and interquartile range (IQR). Pain VAS was compared using independent samples median test and Mann-Whitney U test. Secondary outcomes were analysed using two-tailed Fisher's exact probability testing. Data were analysed using IBM SPSS Statistics software [21].

\section{Results}

Forty participants were assessed for eligibility, five of which could not attend the vaccination clinic due to flu-like symptoms or recent travel from high risk COVID-19 areas. Ideally the flu vaccination should be timed such that peak protection is afforded at the peak of the flu season. Due to COVID-19 restrictions and the uncertainty of lockdowns, the flu clinic was delayed and peak protection would have fallen outside the usual flu season, therefore the study was unable to be continued until the sample size was met. Thirty-five patients were randomised with an allocation ratio of $1: 1$ and included in the final analysis: 19 in the intervention group (ice packs) and 16 in the control group (cold packs) (Figure 1). No patients were lost to follow up, and intention-to-treat and per protocol analysis were equivalent. There were no protocol violations.

There were no differences at baseline between the intervention and control groups (Table 1).

Table 1. Baseline characteristics for participants included in the final analysis.

\begin{tabular}{ccc}
\hline Characteristic & $\begin{array}{c}\text { Intervention Group } \\
\boldsymbol{n}=\mathbf{1 9}(\mathbf{\%})\end{array}$ & $\begin{array}{c}\text { Control Group } \\
\boldsymbol{n}=\mathbf{1 6}(\mathbf{\%})\end{array}$ \\
\hline Median age in years (IQR) & $23.00(3)$ & $22.50(1)$ \\
\hline Female & Gender & $12(75 \%)$ \\
\hline Male & $8(42.1 \%)$ & $4(25 \%)$ \\
\hline $\begin{array}{c}\text { Aboriginal and Torres Strait } \\
\text { Islander }\end{array}$ & $11(57.9 \%)$ & 0 \\
\hline Caucasian & 0 & $7(43.8 \%)$ \\
\hline Othnicity & $9(47.4 \%)$ & $9(56.3 \%)$ \\
\hline
\end{tabular}




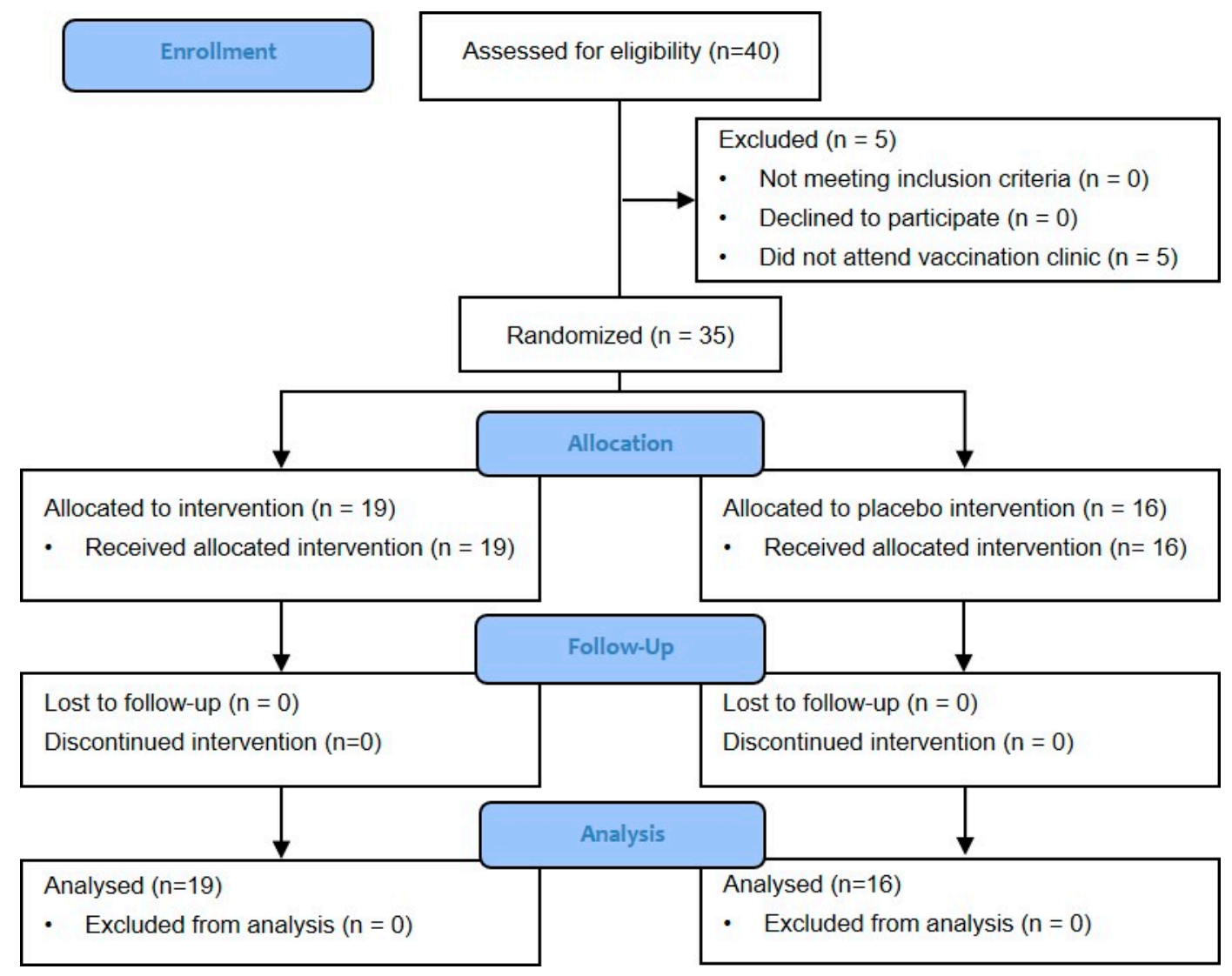

Figure 1. CONSORT flow diagram.

\subsection{Primary Outcome}

Data were not normally distributed ( $p=0.001$, two-tailed Shapiro-Wilk test). The distribution of pain scale scores in both groups were positively skewed (intervention group $=0.55$; control group $=1.32$ ). As demonstrated in Table 2 , the median pain VAS scores did not differ between the groups (intervention group: median pain VAS = 7.0, $\mathrm{IQR}=18$; control group: median pain VAS $=11.0, \mathrm{IQR}=14 ; p=0.26$, two-tailed Mann-Whitney U test).

Table 2. Immediate post-immunisation pain scored on VAS.

\begin{tabular}{cccc}
\hline Pain VAS (cm) & $\begin{array}{c}\text { Intervention Group } \\
(\boldsymbol{n}=\mathbf{1 9 )}\end{array}$ & $\begin{array}{c}\text { Control Group } \\
(\boldsymbol{n}=\mathbf{1 6})\end{array}$ & $p$-Value \\
\hline Median (min-max) & $7(0-26)$ & $11(0-48)$ & 0.26 \\
\hline IQR & 18 & 14 & \\
\hline
\end{tabular}

\subsection{Secondary Outcomes}

Localised pain $24 \mathrm{~h}$ post-immunisation was experienced by $42.1 \%$ in the intervention group and $68.8 \%$ in the control group ( $p=0.18$, two-tailed Fisher's exact probability test). Localised swelling was reported by $21.1 \%$ in the intervention group and $12.5 \%$ in the control group ( $p=0.67$, two-tailed Fisher's exact probability test). Of those in the control group, $18.8 \%$ reported bruising and $6.3 \%$ reported erythema while neither of these adverse events were reported in the intervention group (Table 3 ). 
Table 3. Adverse effects $24 \mathrm{~h}$ post-immunisation.

\begin{tabular}{cccc}
\hline Adverse Event & $\begin{array}{c}\text { Intervention Group } \\
\mathbf{N = 1 9} \boldsymbol{n} \mathbf{( \% )}\end{array}$ & $\begin{array}{c}\text { Control Group } \\
\mathbf{N = 1 6} \boldsymbol{n} \mathbf{( \% )}\end{array}$ & $p$-Value \\
\hline Localised to site of injection & \\
\hline Pain & $8(42.1 \%)$ & $11(68.8 \%)$ & 0.18 \\
\hline Swelling & $4(21.1 \%)$ & $2(12.5 \%)$ & 0.67 \\
\hline Bruising & $0(0 \%)$ & $3(18.8 \%)$ & 0.09 \\
\hline Redness & $0(0 \%)$ & $1(6.3 \%)$ & 0.46 \\
\hline Itching & $0(0 \%)$ & $0(0 \%)$ & N/A \\
\hline Anaphylaxis & $0(0 \%)$ & Systemic & N/A \\
\hline
\end{tabular}

\section{Discussion}

Patients who received ice packs reported lower median pain scores compared to those given cold packs, however the results were not significant, potentially due to under powering of the study. There was no difference in adverse effects such as redness, swelling, bruising of the skin or prolonged pain, localised to the site of injection.

The subjective nature of pain makes it difficult to measure [22]. Individual characteristics including age, sex, past medical history and previous pain experiences are known to affect the perception of pain [22]. Medical students were recruited as participants because of convenience in organising the study around student-driven influenza clinics. As health literate participants, students were more likely to be compliant, and more reliable in the reporting of pain compared with the normal general practice population, however they may also be less likely to be anxious, and their perception of pain may differ as reflected by low VAS scores.

Ice is available in both primary care and hospital settings and has a quick onset of action, approximately 30s [12]. In addition, compared to topical local anaesthetic creams (approximately AUD 10 per dose) and vapocoolant sprays (AUD 0.70 per dose), ice packs (AUD 0.50 per dose) are more cost effective [1,23]. Ice packs have few associated adverse effects. Cold-induced anaphylaxis is the most severe potential side effect of ice, but it is rare and there are currently no conclusive data regarding its incidence [24]. The main disadvantage is their short life span in ambient temperature.

Injectable vaccines, administered according to standard guidelines, often vary in degree of painfulness depending on their composition [25]. In children, pneumococcal conjugate vaccine $(\mathrm{PCV})$ is considered more painful compared to the diphtheria, tetanus and acellular pertussis (DTaP) vaccine and Haemophilus influenza type b (Hib) vaccine [26]. Likewise, concurrent hepatitis A and B vaccinations in adults demonstrated increased levels of pain compared to hepatitis B vaccination alone [27]. The current trend towards increased number of recommended vaccines, further makes the use of readily available and inexpensive ice packs as an analgesic agent attractive to enhance acceptance of vaccine injections.

\section{Limitations}

Compliance with government regulations, implemented during the COVID-19 pandemic, was required to ensure the safety of patients, health professionals and research staff. Some members of our research team and participating GP practices were deployed to other areas, with different responsibilities. Strict travel restrictions prevented travel to one of our clinical research centres in Townsville. As such, recruitment was from a single centre in Mackay, and our sample size was considerably reduced. Government restrictions permitted a maximum of 10 people in the participating general practice at one time and several rooms were required to ensure adequate social distancing. To comply with quarantine guidelines, students were advised not to attend the clinic if they were symptomatic or had recently 
travelled to high-risk COVID-19 locations. Our final sample size of 35 participants fell short of the 90 required to reach the desired study power, thereby limiting the potential to detect if ice packs were able to reduce pain associated with vaccine injection. Another limitation of this study was that participants were not asked to report their fear of needles from past experiences as these could potentially impact the pain ratings for the current injection. Given the relatively low VAS scores for vaccination-related pain in this study, it is unlikely that the variable of anxiety from previous vaccinations impacted the results.

\section{Conclusions}

We hypothesised that pain scores would be lower with the use of ice; however, results were inconclusive, potentially due to under-powering. Future studies investigating the efficacy of ice in reducing vaccine-related pain with larger sample sizes could potentially inform current practice.

Author Contributions: Conceptualization, C.H.; formal analysis, Y.E.; investigation, Y.E.; resources, Y.E.; data curation, Y.E.; writing — original draft preparation, Y.E.; writing—review and editing, L.H.; supervision, C.H. and J.B.; All authors have read and agreed to the published version of the manuscript.

Funding: The study was supported by James Cook University. The funder played no role in the design, collection, analysis and interpretation of data, or the decision to submit results.

Institutional Review Board Statement: The study was conducted according to the guidelines of the Declaration of Helsinki, and approved by the James Cook University Human Research Ethics committee (H7871).

Informed Consent Statement: Informed consent was obtained from all subjects involved in the study.

Acknowledgments: The research team would like to thank Michael Wohlfahrt, Annaliese Wohlfarht, Mia Browning and Mackay City Medical practice for their contributions to this study.

Conflicts of Interest: None of the authors have any conflict of interest to declare.

\section{References}

1. Mawhorter, S.; Daugherty, L.; Ford, A.; Hughes, R.; Metzger, D.; Easley, K. Topical vapocoolant quickly and effectively reduces vaccine-associated pain: Results of a randomized, single-blinded, placebo-controlled study. J. Travel Med. 2004, 11, $267-272$. [CrossRef] [PubMed]

2. The Burden of Vaccine Preventable Diseases in Australia, Table of Contents-Australian Institute of Health and Welfare. Australian Institute of Health and Welfare. Available online: https:/ / www.aihw.gov.au/reports/immunisation/the-burden-of-vaccinepreventable-diseases / contents / table-of-contents (accessed on 21 June 2020).

3. Nir, Y.; Paz, A.; Sabo, E.; Potasman, I. Fear of injections in young adults: Prevalence and associations. Am. J. Trop. Med. Hyg. 2003, 68, 341-344. [CrossRef] [PubMed]

4. Bienvenu, O.J.; Eaton, W.W. The epidemiology of blood-injection-injury phobia. Psychol. Med. 1998, 28, 1129-1136. [CrossRef] [PubMed]

5. Hart, P.S.; Yanny, W. Needle phobia and malignant vasovagal syndrome. Anaesthesia 1998, 53, 1002-1004. [CrossRef] [PubMed]

6. Gidudu, J.F.; Walco, G.A.; Taddio, A.; Zempsky, W.T.; Halperin, S.A.; Calugar, A.; Gibbs, N.A.; Hennig, R.; Jovancevic, M.; Netterlid, E.; et al. Immunization site pain: Case definition and guidelines for collection, analysis, and presentation of immunization safety data. Vaccine 2012, 30, 4558-4577. [CrossRef] [PubMed]

7. Melzack, R.; Wall, P.D. Pain mechanisms: A new theory. Science 1965, 150, 971-979. [CrossRef] [PubMed]

8. Kunesch, E.; Schmidt, R.; Nordin, M.; Wallin, U.; Hagbarth, K.E. Peripheral neural correlates of cutaneous anaesthesia induced by skin cooling in man. Acta Physiol. Scand. 1987, 129, 247-257. [CrossRef] [PubMed]

9. Schoenfeld, A.D.; Lox, C.D.; Chen, C.H.; Lutherer, L.O. Pain threshold changes induced by acute exposure to altered ambient temperatures. Peptides 1985, 6 (Suppl. 1), 19-22. [CrossRef]

10. Breslin, M.; Lam, P.; Murrell, G.A. Acute effects of cold therapy on knee skin surface temperature: Gel pack versus ice bag. BMJ Open Sport Exerc. Med. 2015, 1, e000037. [CrossRef] [PubMed]

11. Cameron, M. Physical Agents. In Rehabilitation, 4th ed.; Elsevier-Health Sciences Division: Amsterdam, The Netherlands, 2017; pp. 129-144.

12. Akcimen, M.; Bedel, C.; Selvi, F. Application of ice and vapocoolant spray to reduce tetanus vaccine pain: A prospective, randomized, controlled, clinical study. Ann. Med Res. 2019, 26, 995-998. [CrossRef] 
13. Hall, L.M.; Ediriweera, Y.; Banks, J.; Nambiar, A.; Heal, C. Cooling to reduce the pain associated with vaccination: A systematic review. Vaccine 2020, 38, 8082-8089. [CrossRef] [PubMed]

14. Ediriweera, Y.; Banks, J.; Hall, L.; Heal, C. A study protocol for a randomised controlled trial of ice to reduce the pain of immunisation-the ICE trial. Aust. J. Gen. Pract. 2021, in press.

15. RACGP-6.1 Immunisation. Racgp.org.au. Available online: https:/ /www.racgp.org.au/clinical-resources/clinical-guidelines / key-racgp-guidelines/view-all-racgp-guidelines/guidelines-for-preventive-activities-in-general-pr/preventive-activities-inolder-age/immunisationPublished2020 (accessed on 24 June 2020).

16. RACGP-Influenza Prevention. Racgp.org.au. Available online: https://www.racgp.org.au/clinical-resources/clinical-guidelines / key-racgp-guidelines/view-all-racgp-guidelines/national-guide/chapter-9-respiratory-health/influenza-prevention (accessed on 24 June 2020).

17. Taddio, A.; Lord, A.; Hogan, M.E.; Kikuta, A.; Yiu, A.; Darra, E.; Bruinse, B.; Keogh, T.; Stephens, D. A randomized controlled trial of analgesia during vaccination in adults. Vaccine 2010, 28, 5365-5369. [CrossRef]

18. Todd, K.H.; Funk, K.G.; Funk, J.P.; Bonacci, R. Clinical significance of reported changes in pain severity. Ann. Emerg. Med. 1996, 27, 485-489. [CrossRef]

19. Cohen, M.; Wolfe, R.; Mai, T.; Lewis, D. A randomized, double blind, placebo controlled trial of a topical cream containing glucosamine sulfate, chondroitin sulfate, and camphor for osteoarthritis of the knee. J. Rheumatol. 2003, 30, 523-528.

20. Hawker, G.A.; Mian, S.; Kendzerska, T.; French, M. Measures of adult pain: Visual Analog Scale for Pain (VAS Pain), Numeric Rating Scale for Pain (NRS Pain), McGill Pain Questionnaire (MPQ), Short-Form McGill Pain Questionnaire (SF-MPQ), Chronic Pain Grade Scale (CPGS), Short Form-36 Bodily Pain Scale (SF-36 BPS), and Measure of Intermittent and Constant Osteoarthritis Pain (ICOAP). Arthritis Care Res. 2011, 63 (Suppl. 11), S240-S252.

21. IBM Corp. IBM SPSS Statistics for Windows; Version 26.0; IBM Corp: Armonk, NY, USA, 2019.

22. Shankland, W.E., 2nd. Factors that affect pain behavior. Cranio 2011, 29, 144-154. [CrossRef] [PubMed]

23. Medi-Ice Pak Mini Ice Cells-32 per Sheet. Alphafirstaid.com.au. Available online: https://www.alphafirstaid.com.au/product/ mini-ice-cells-medi-ice-pak-32-per-sheet-single-use (accessed on 3 July 2020).

24. Benelli, E.; Longo, G.; Barbi, E.; Berti, I. Anaphylaxis in atypical cold urticaria: Case report and review of literature. Ital. J. Pediatr. 2018, 44, 135. [CrossRef] [PubMed]

25. The Weekly Epidemiological Record (WER). World Health Organization. Available online: https://www.who.int/wer/en/ (accessed on 2 July 2020).

26. Ipp, M.; Parkin, P.C.; Lear, N.; Goldbach, M.; Taddio, A. Order of vaccine injection and infant pain response. Arch. Pediatr. Adolesc. Med. 2009, 163, 469-472. [CrossRef]

27. Bryan, J.P.; McCardle, P.; South-Paul, J.E.; Fogarty, J.P.; Legters, L.J.; Perine, P.L. Randomized controlled trial of concurrent hepatitis A and B vaccination. Mil Med. 2001, 166, 95-101. [CrossRef] 